\title{
AMS Results on the Properties of the Fluxes of Elementary Particles and Nuclei in Primary Cosmic Rays
}

\author{
Andrei Kounine ${ }^{* \dagger}$ \\ Massachusetts Institute of Technology, Cambridge, USA \\ E-mail: Andrei.Kouninedcern.ch
}

Precision measurements by AMS of the fluxes of cosmic ray positrons, electrons, antiprotons, protons and light nuclei as well as their rations reveal several unexpected and intriguing features. The presented measurements extend the energy range of the previous observations with much increased precision. The new results show that the behavior of fluxes and electrons and positrons at around $300 \mathrm{GeV}$ is consistent with a new source that produce equal amount of high energy electrons and positrons. Surprisingly, in this rigidity range the spectral indices of cosmic ray nuclei, including protons and helium, experience progressive hardening over the rigidity interval of few hundred GV. In addition, in the absolute rigidity range $60-500 \mathrm{GV}$, the antiproton, proton, and positron fluxes are found to have nearly identical rigidity dependence and the electron flux exhibits different rigidity dependence. The measured ratio of proton-to-Helium fluxes is not constant at high rigidities, whereas the ratios of other primary cosmic ray nuclei such as carbon and oxygen to helium are constant. From the behavior of the flux ratio of beryllium to carbon the age of cosmic rays in the galaxy is found to be $\sim 12$ million years, and, remarkably, the measured boron-to-carbon flux ratio is found to follow a single power law, consistent with the Kolmogorov turbulence model of magnetized plasma. Most importantly, AMS continues studies of complex antimatter candidates with stringent detector verification and collection of additional data.

35th International Cosmic Ray Conference - ICRC2017

10-20 July, 2017

Bexco, Busan, Korea

*Speaker.

${ }^{\dagger}$ On Behalf of the AMS Collaboration. 


\section{AMS Detector}

The AMS Experiment is the most powerful physics detector of charged cosmic rays ever deployed in space and is exploring a new and exciting frontier in physics research. As a magnetic spectrometer, AMS is unique in physics research as it studies charged particles and nuclei from original sources in the cosmos. It was installed on the International Space Station, ISS, on 19 May 2011 to conduct a long duration mission of fundamental physics research in space for understanding of dark matter and complex antimatter in the cosmos, the properties of primary and secondary cosmic rays as well as for discovery of new, unexpected phenomena. The improvement in accuracy over previous measurements is made possible through its long duration time in space, large acceptance, built in redundant systems and its thorough calibration in the CERN test beam. Since its installation on the ISS in May 2011, AMS has collected more than 100 billion cosmic rays.

The layout of the AMS detector [1] is shown in Fig. 1. It consists of 9 planes of precision silicon Tracker; a Transition Radiation Detector, TRD; four planes of Time of Flight counters, TOF; a Magnet; an array of anti-coincidence counters, ACC, surrounding the inner Tracker; a Ring Imaging Čerenkov detector, RICH; and an Electromagnetic Calorimeter, ECAL. The figure also shows a high energy positron of $868 \mathrm{GeV}$ recorded by AMS.

Together, the tracker and the magnet measure charged cosmic rays with momentum $p$, charge $Z$ and rigidity $R=p c / Z e$. The tracker [2] has nine layers, the first at the top of the detector, the second just above the magnet, six within the bore of the magnet, and the last just above the ECAL. Each layer contains double-sided silicon microstrip detectors that independently measure the $x$ and

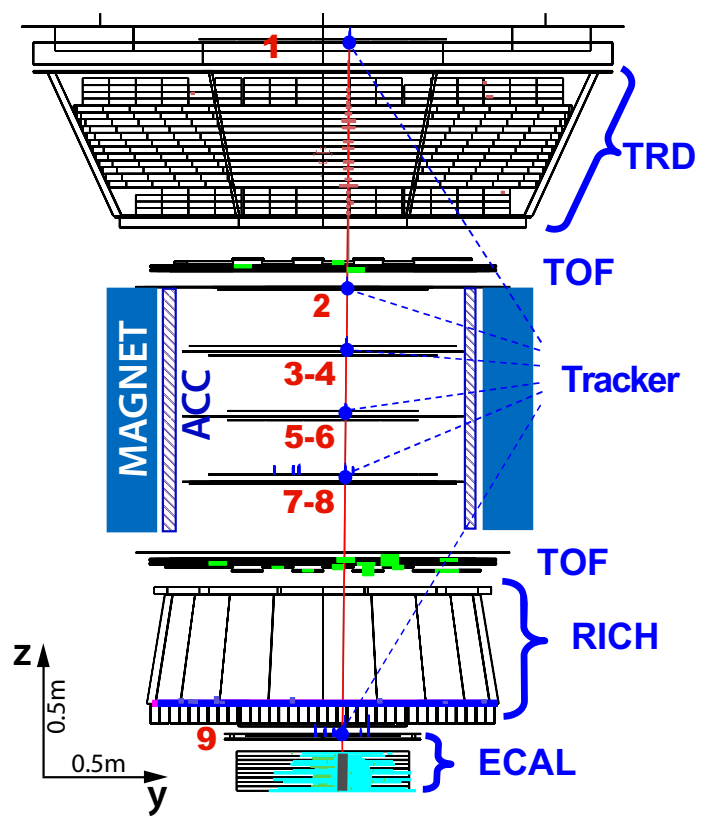

Figure 1: A $868 \mathrm{GeV}$ positron event as measured by the AMS detector on the ISS in the (y-z) plane. Tracker planes 1-9 measure the particle charge, sign and momentum. The TRD identifies the particle as an electron/positron. The TOF measures the charge and ensures that the particle is downward-going. The RICH measures the charge and velocity. The ECAL independently identifies the particle as an electron/positron and measures its energy. 
$y$ coordinates. The tracker accurately determines the particle trajectory by multiple measurements of the coordinates with a resolution in each layer of $10 \mu \mathrm{m}$ in the bending $(y)$ direction. The maximum detectable rigidity, MDR, is $2 \mathrm{TV}$ for $|Z|=1,3.2 \mathrm{TV}$ for helium and $3.7 \mathrm{TV}$ for carbon nuclei over the $3 \mathrm{~m}$ lever arm. Signal amplitude from each layer of the tracker also provides an independent measurement of charge $|Z|$. The overall charge resolution of the tracker is $\Delta Z / Z$ is $5 \%$ for $|Z|=1,3.5 \%$ for helium and $2 \%$ for carbon nuclei.

The TOF counters measure the velocity $\beta=v / c$ and $|Z|$ of cosmic rays. Two TOF planes are located above and two planes are located below the magnet [3]. For $|Z|=1$ particles, the average time resolution of each counter has been measured to be $160 \mathrm{ps}$ and the overall velocity resolution to be $\Delta \beta / \beta^{2}=4 \%$. The time resolution improves for higher charges to reach $50 \mathrm{ps}$ for oxygen nuclei with the corresponding improvement in the velocity resolution of $\Delta \beta / \beta^{2}=1 \%$. This discriminates between downward- and upward-going particles. The coincidence of signals from the four TOF planes provides a basis for charged particle trigger, whereas the coincidence of 3 out of the 4 TOF layers provides an unbiased trigger. The unbiased trigger, prescaled to $1 \%$, is used to measure the trigger efficiency from the data.

To distinguish antiprotons and positrons from protons and electrons which are reconstructed in the tracker with wrong rigidity sign due to the finite tracker resolution or due to interactions with the detector materials, a charge confusion estimator $\Lambda_{\mathrm{CC}}$ is defined using the boosted decision tree technique [4]. The estimator combines information from the tracker such as the track $\chi^{2} / d$.f., rigidities reconstructed with different combination of tracker layers, the number of hits in the vicinity of the track, and the charge measurements in the TOF and the tracker. With this method, antiprotons/positrons have $\Lambda_{\mathrm{CC}} \sim+1$ whereas charge confusion protons/electrons have $\Lambda_{\mathrm{CC}} \sim-1$. This ensures efficient separation of the signal from the charge confusion events.

The TRD uses transition radiation to distinguish between $\bar{p}(p)$ and $e^{-}\left(e^{+}\right)$and $d E / d x$ to independently identify nuclei [5]. It consists of 5248 proportional tubes of $6 \mathrm{~mm}$ diameter with a maximum length of $2 \mathrm{~m}$ assembled in 16-tube modules. The 328 modules are mounted in 20 layers. There are 12 layers of proportional tubes along the $y$ axis located in the middle of the TRD
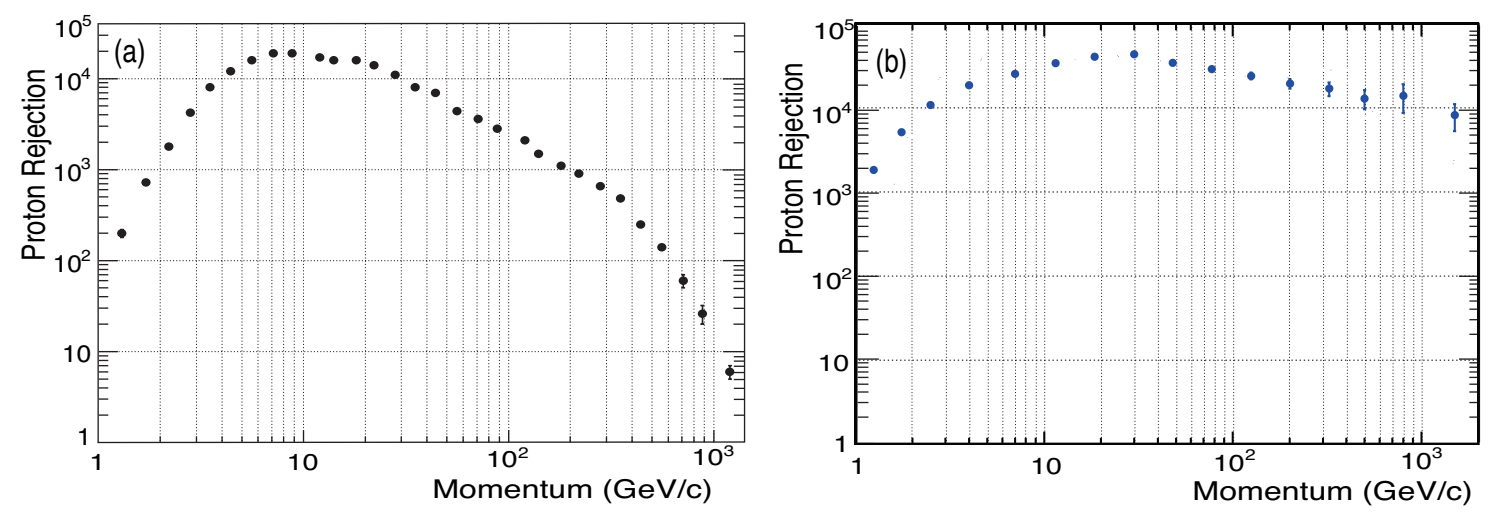

Figure 2: (a) The proton rejection measured by the TRD as a function of track momentum at $90 \%$ selection efficiency for $\mathrm{e}^{ \pm}$. (b) The measured proton rejection using the ECAL and the Tracker. For $90 \% \mathrm{e}^{ \pm}$ECAL selection efficiency, the measured proton rejection is $\sim 10,000$ for the combination of the ECAL and the Tracker in the momentum range $3-500 \mathrm{GeV} / \mathrm{c}$, independent of the TRD. 
and, along the $x$ axis, four layers located on top and four on the bottom. To differentiate between $\bar{p}(p)$ and $e^{-}\left(e^{+}\right)$in the TRD, signals from the 20 layers of proportional tubes are combined into a TRD estimator $\Lambda_{\mathrm{TRD}}$ formed from the ratio of the log-likelihood probability of the $e^{ \pm}$hypothesis to that of the $\bar{p}(p)$ hypothesis in each layer [6]. Antiprotons and protons have $\Lambda_{\mathrm{TRD}} \sim 1$ whereas electrons and positrons have $\Lambda_{\mathrm{TRD}} \sim 0.5$. This provides efficient separation of $\bar{p}(p)$ and $e^{-}\left(e^{+}\right)$. For illustration, the proton rejection power of the estimator at $90 \% \mathrm{e}^{ \pm}$efficiency is shown in Fig. 2a as a function of energy. It reaches up to $10^{4}$ in the energy range of interest.

The ring imaging Čerenkov detector [7] measures velocity and $|Z|$. It consists of two radiators, an expansion volume, and a photodetection plane. The dielectric radiators induce the emission of a cone of Čerenkov photons when traversed by charged particles with a velocity greater than the velocity of light in the material. The central radiator is formed by 16 sodium fluoride, NaF, tiles, each $85 \times 85 \times 5 \mathrm{~mm}^{3}$, with a refractive index $n=1.33$. These are surrounded by 92 tiles, each $115 \times 115 \times 25 \mathrm{~mm}^{3}$, of silica aerogel with a refractive index $n=1.05$. This allows the detection of particles with velocities $\beta>0.75$ with the $\mathrm{NaF}$ radiator and $\beta>0.953$ with the aerogel radiator. The expansion volume has a distance along $z$ of $470 \mathrm{~mm}$ and is surrounded by a high reflectivity mirror to increase detection efficiency. The photodetection plane is an array of 10880 photosensors with an effective spatial granularity of $8.5 \times 8.5 \mathrm{~mm}^{2}$. The sum of the signal amplitudes is proportional to $Z^{2}$. The opening angle of the Čerenkov radiation cone is a measure of the velocity of the incoming charged particle. Typical velocity resolution is $\Delta \beta / \beta=0.1 \%$ for $|Z|=1$.

The three-dimensional imaging capability of the 17 radiation length ECAL [8] allows for an accurate measurement of the $e^{ \pm}$energy $E$ and shower shape. It consists of a multilayer sandwich of 98 lead foils and $\sim 50,000$ scintillating fibers with an active area of $648 \times 648 \mathrm{~mm}^{2}$ and a thickness of $166.5 \mathrm{~mm}$. The calorimeter is composed of 9 superlayers, with the fibers running in one direction only in each superlayer. The 3-D imaging capability of the detector is obtained by stacking alternate superlayers with fibers parallel to the $x$ - and $y$-axes ( 5 and 4 superlayers, respectively). The energy resolution has been measured to be $\sigma(E) / E=\sqrt{(0.104)^{2} / E+(0.014)^{2}}(E$ in GeV). The uncertainty of the absolute $e^{ \pm}$energy scale has been verified to be $2 \%$ in the range $10-290 \mathrm{GeV}$. Below $10 \mathrm{GeV}$ it increases to $5 \%$ at $0.5 \mathrm{GeV}$ and above $290 \mathrm{GeV}$ to $4 \%$ at $700 \mathrm{GeV}$. To cleanly separate protons from electrons and positrons, an ECAL estimator[8], is constructed using the 3-D shower shape in the ECAL. The proton rejection power of the ECAL estimator when combined with the energy-momentum matching requirement $E / p>0.75$ reaches $\sim 10,000$ (see Fig. 2b).

Before launch, AMS was extensively calibrated at the CERN SPS with 180 and $400 \mathrm{GeV} / c$ proton beams and positron, electron, and pion beams of 10 to $290 \mathrm{GeV} / c$. In total, calibrations with 18 different energies and particles at 2000 positions were performed. These data allow the determination of the tracker rigidity resolution function with high precision and the verification of the absolute rigidity scale. Since launch, the detector has been monitored and controlled around the clock. Its performance has been steady over time.

Monte Carlo simulated events are produced using a dedicated program developed by the collaboration from the GEANT 4.10.1 package [9]. This program simulates electromagnetic and hadronic interactions of particles in the material of AMS and generates detector responses. The digitization of the signals is simulated precisely according to the measured characteristics of the electronics. The simulated events then undergo the same reconstruction as used for the data. 


\section{Precision measurement of the positron fraction and fluxes of $\mathrm{e}^{+}$and $\mathrm{e}^{-}$}

Over the past few decades there has been strong interest in the cosmic ray positron fraction in both particle physics and astrophysics [10]. It was considered as a sensitive probe into the properties of Dark Matter in our Galaxy. The first experimental excitement came from the HEAT experiment which was the first to report "small positron flux of nonstandard origin above $5 \mathrm{GeV}$ " [11]. This deviation of the behavior of the positron fraction from the one expected from the positron production in collisions of ordinary cosmic rays was then confirmed by AMS-01 [12], PAMELA [13] and Fermi-LAT [14] experiments. Precision AMS results [6, 15] on the positron fraction not only clearly show the rise of the positron fraction above its minimum at $7.8 \mathrm{GeV}$, but also existence of a maximum at $265 \pm 22 \mathrm{GeV}[15]$.

Much more information on the dynamics of this behavior is in the measurements of the individual fluxes of electrons and positrons [16]. AMS has observed that with a data set of 20 million electrons and positrons [17], the electron flux and positron flux display different behaviors in their magnitude and energy dependence (see Fig. 3 (left plot)). The most striking feature is in the hardening of the positron spectrum above $30 \mathrm{GeV}$, seen as the progressive rise of the positron spectrum. This may be interpreted as a contribution of a new, yet unknown, physics source.

To quantitatively examine the energy dependence of the fluxes in a model independent way, each of them is fit with a spectral index, $\gamma_{e^{ \pm}}=d\left(\log \left(\Phi_{e^{ \pm}}\right)\right) / d(\log (E))$ over a sliding energy window, where the width of the window varies with energy to have sufficient sensitivity to determine the spectral index. The resulting energy dependencies of the fitted spectral indices are shown in Fig. 3 (right plot). The steep decreases of the spectral indices below $10 \mathrm{GeV}$ are due to solar modulation. Above $20 \mathrm{GeV}$, that is, above the effects of solar modulation, the spectral indices for positrons and electrons are significantly different. From 20 to $200 \mathrm{GeV}, \gamma_{e^{+}}$is significantly higher than $\gamma_{e^{-}}$. This demonstrates that the increase with energy observed in the positron fraction is,

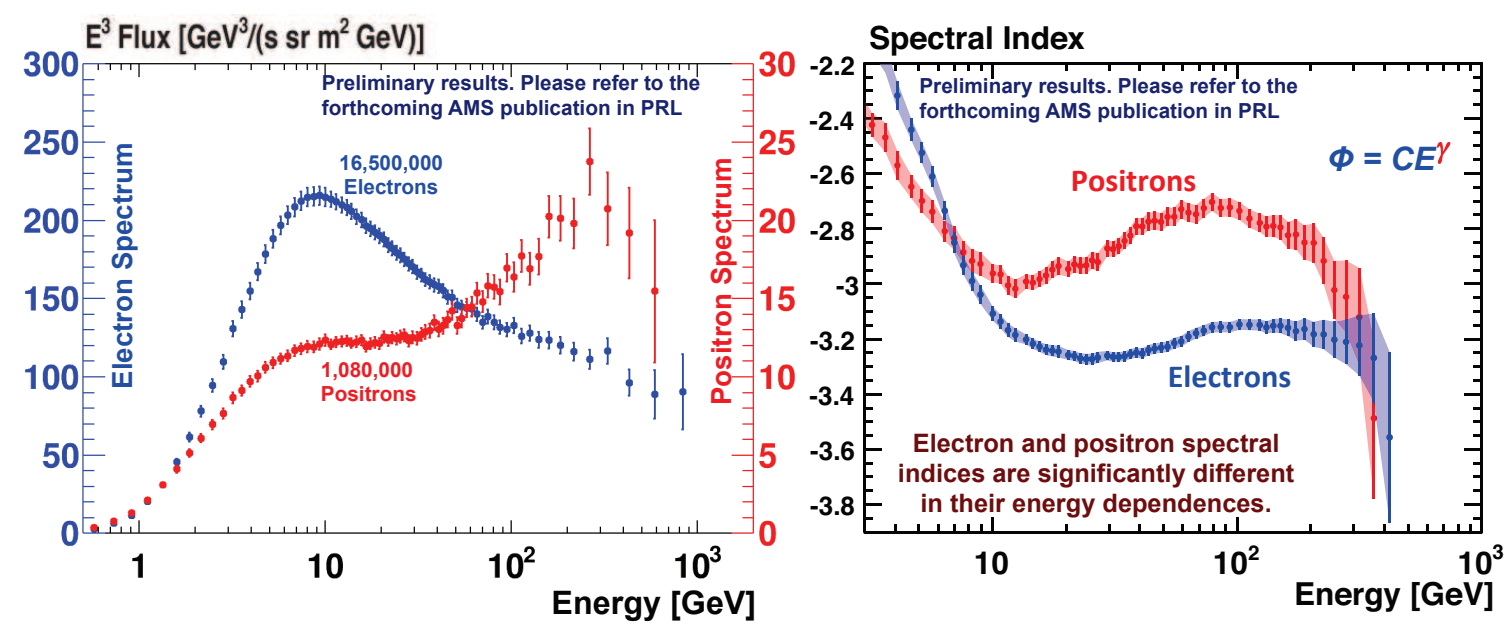

Figure 3: (left plot)The AMS positron and electron fluxes multiplied by $E^{3}$. The present measurement extends the energy range to $700 \mathrm{GeV}$ for positrons and to $1000 \mathrm{GeV}$ for electrons. The electron flux and the positron flux are different in their magnitude and energy dependence. (right plot) The spectral indices of the electron flux $\gamma_{e^{-}}$and the positron flux $\gamma_{e^{+}}$as a function of energy. 

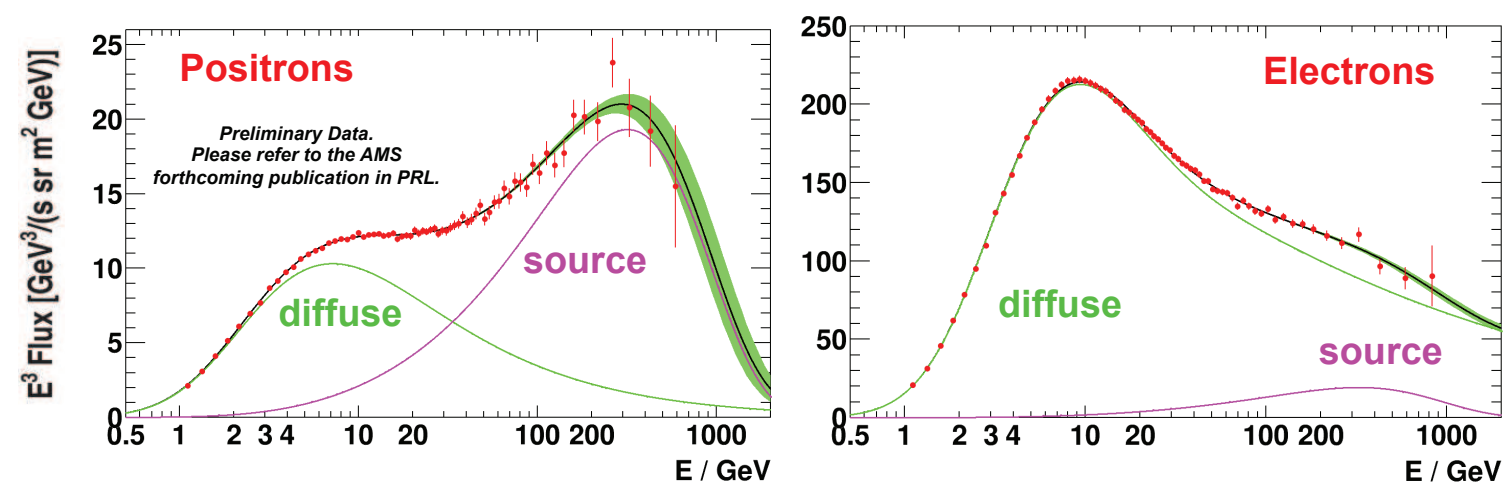

Figure 4: Fluxes of positrons and electrons in comparison with the Minimal Model which assumes common source term for the $e^{+}$and $e^{-}$fluxes show its equal contribution both to positrons and electrons.

indeed, due to an increase in the positron flux and not to a loss in the electron flux.

There has been much speculation over the last few decades on the possible contribution of heavy dark matter particles to the positron spectrum. When particles of dark matter collide, they produce equal amounts of energetic electrons and positrons, which may create structures in the corresponding spectra: an increase with energy followed by a sharp drop off at the mass of dark matter as well as an isotropic distribution of the arrival directions. The expected effect is less pronounced in the abundant cosmic rays like electrons, and enhanced in rare species like positrons - exactly as observed by AMS.

A Minimal Model introduced in Ref. [6] allows to highlight these features of $e^{+}$and $e^{-}$fluxes. In this model the $e^{+}$and $e^{-}$fluxes, are parametrized as the sum of individual diffuse power law spectra and the contribution of a single common source of $e^{ \pm}$with an exponential cutoff parameter, $E_{s}$, for the source term: $\Phi_{e^{ \pm}}=C_{e^{ \pm}} E^{-\gamma_{e} m m}+C_{s} E^{-\gamma_{s}} e^{-E / E_{s}}$. The fit results is shown in Fig. 4. The agreement between the data and the model shows that that the source of high energy electrons and positrons contributes equally to both electron and positron fluxes.

All the observed features of $e^{+}$and $e^{-}$fluxes are well consistent with the Dark Matter models with mass of $\sim 1 \mathrm{TeV}$, as shown in Fig. 5 (left plot). As seen, after rising from $8 \mathrm{GeV}$ above the rate
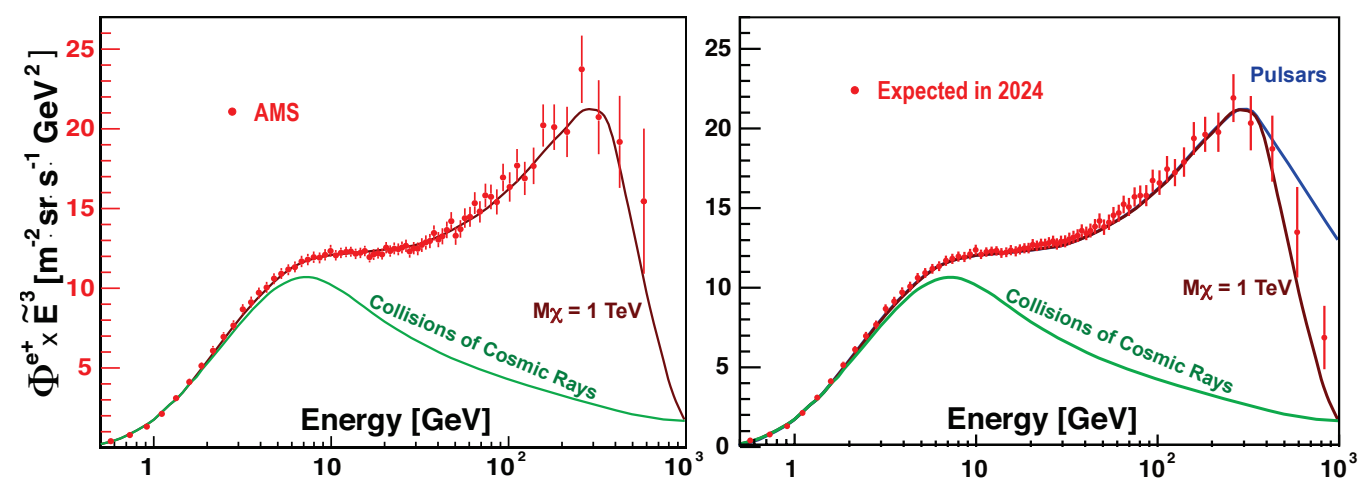

Figure 5: (left plot) The measured positron flux in comparison with the 1 Tev Dark Matter model. (right plot) Expected the energy reach and accuracy for the positron flux measurement by 2024 . 
expected from cosmic ray collisions with Interstellar Matter (ISM), shown as the green curve, the measured spectrum exhibits a tendency to drop off sharply at high energies. With more data AMS will explore this very intriguing feature of the positron flux at high energies. Fig. 5 (right plot) shows the energy reach and accuracy of AMS by 2024 (i.e. expected ISS lifetime).

Following the publication of our papers $[6,16]$, there have been many interpretations [18] with two popular classes. In the first, the excess of $e^{+}$comes from pulsars. In this case, after flattening out with energy the positron fraction will begin to slowly decrease and a dipole anisotropy should be observed. In the second, the shape of the positron fraction is due to dark matter collisions. In this case, after flattening out, the fraction will decrease rapidly with energy due to the finite and specific mass of the dark matter particle (see Fig. 5) and no dipole anisotropy will be observed. Over its lifetime, AMS will reach a dipole anisotropy sensitivity of $\delta \simeq 0.01$ at the $95 \%$ C.L. [19].

\section{Properties of fluxes of elementary particles and their ratios}

The AMS detector comprises seven instruments, which independently identify different elementary particles as well as nuclei. Protons, helium, lithium, carbon, oxygen and heavier nuclei up to iron are intensively studied by AMS (see Fig. 6).

Protons are the most abundant particles in cosmic rays. It has been traditionally assumed for decades that the spectrum of cosmic ray protons can be described by a single power law function (see for instance the current PDG Chapter "29. COSMIC RAYS"). It was the CREAM experiment to point out first that to reconcile the AMS-01 proton spectrum measurements below $200 \mathrm{GeV}$ [20] (well described by a single power law function) with their own measurement in the range from $2.5 \mathrm{TeV}$ to $250 \mathrm{TeV}$ [21] (also well described with a single power law function) a change of the spectral index at few hundred $\mathrm{GeV}$ is required. However to study the detailed behavior of this transition required AMS precision [22, 23]. Fig. 7 shows the AMS results on proton proton flux as a function of rigidity (i.e. momentum/charge) together with the earlier results of PAMELA. The proton spectrum measured by AMS hardens progressively above $100 \mathrm{GV}$ over the range of

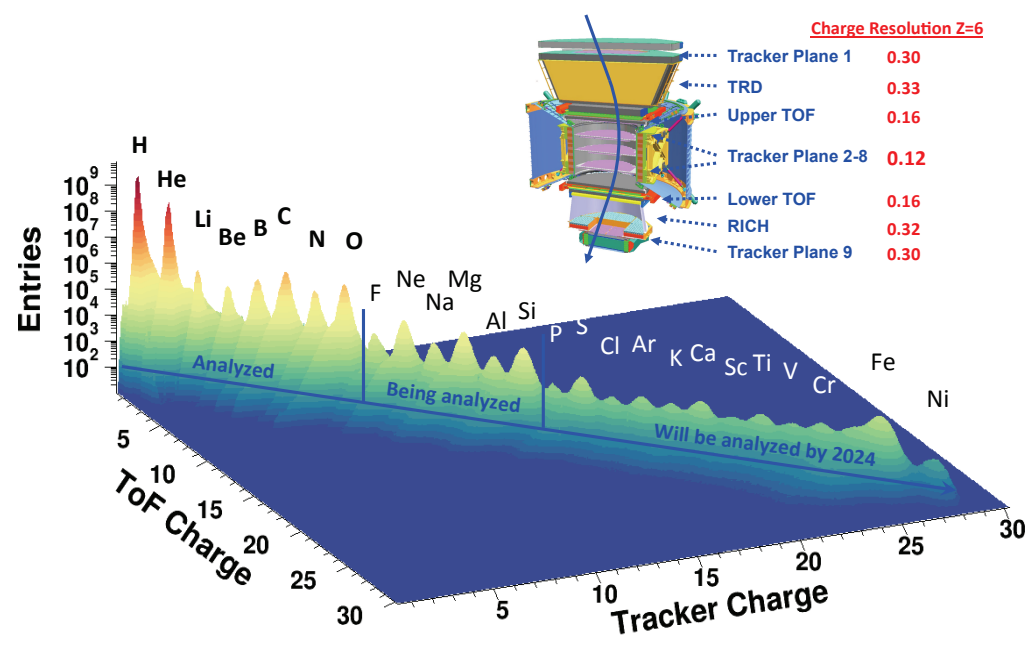

Figure 6: Cosmic ray nuclei studied by AMS. 


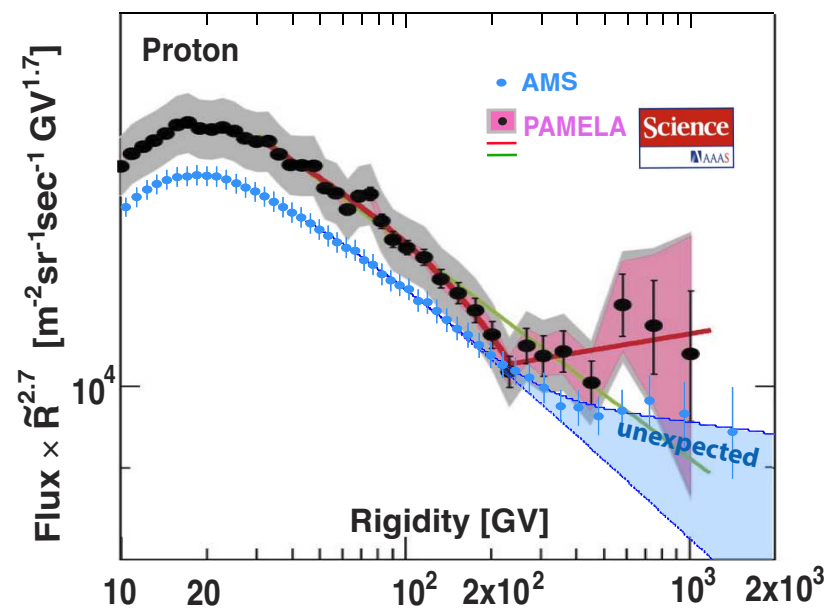

Figure 7: The AMS proton spectrum (blue circles) hardens progressively above $100 \mathrm{GV}$ over the range of few hundred GV as indicated by the blue shaded area on the plot. Abrupt spectral hardening reported by the PAMELA experiment [24] (also shown on the plot) is not supported by the precision AMS measurements.

few hundred GV. This is in direct contradiction with the earlier conclusion by the PAMELA experiment [24] that "At 230 to $240 \mathrm{GV}$, the proton and helium data exhibit an abrupt spectral hardening".

Experimental data on the cosmic ray antiprotons, $\bar{p}$, are crucial for understanding the origin of antiprotons in cosmos and for providing an insight into new physics phenomena. While some of cosmic ray $\bar{p}$ are produced by interactions of the cosmic ray nuclei with the interstellar gas, there could be a substantial contribution from annihilation of dark matter particles, acceleration of antiprotons to high energies in astrophysical objects, or evaporation of primordial black holes. The sensitivity of cosmic ray antiprotons to these new phenomena is complementary to the sensitivity of the measurements of cosmic ray positrons $e^{+}$. However, to measure the antiproton flux to $1 \%$ accuracy requires a separation power of $\sim 10^{6}$. As an example of that, Fig. 8 shows clear separation

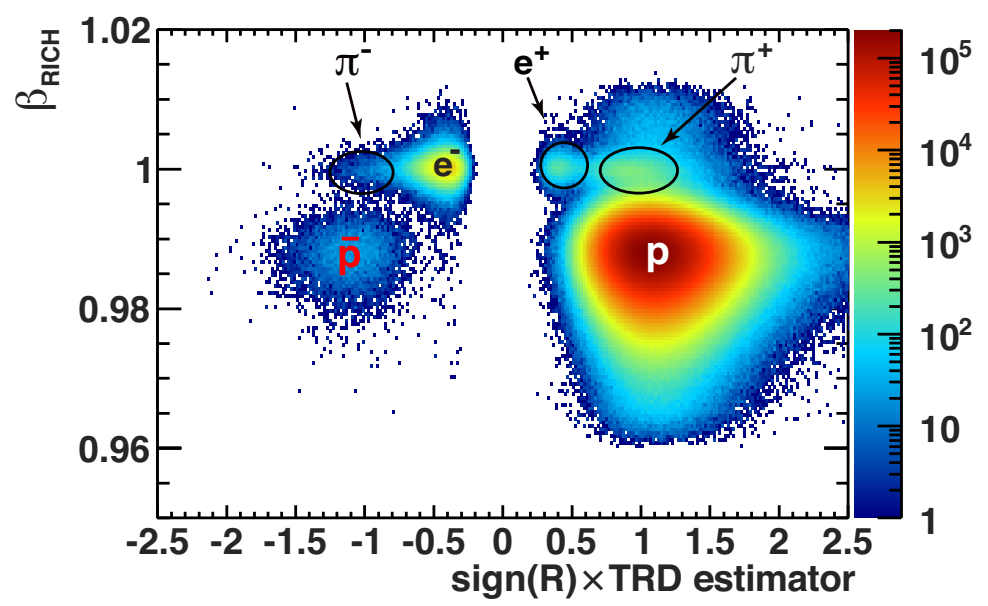

Figure 8: Negative rigidity and positive rigidity data samples in the $\left(\beta_{\mathrm{RICH}}-\operatorname{sign}(R) \times \Lambda_{\mathrm{TRD}}\right)$ plane for the absolute rigidity range 5.4-6.5 GV. The contributions of $\bar{p}, p, e^{+}, e^{-}, \pi^{+}$, and $\pi^{-}$are clearly seen. The antiproton signal is well separated from the backgrounds. 

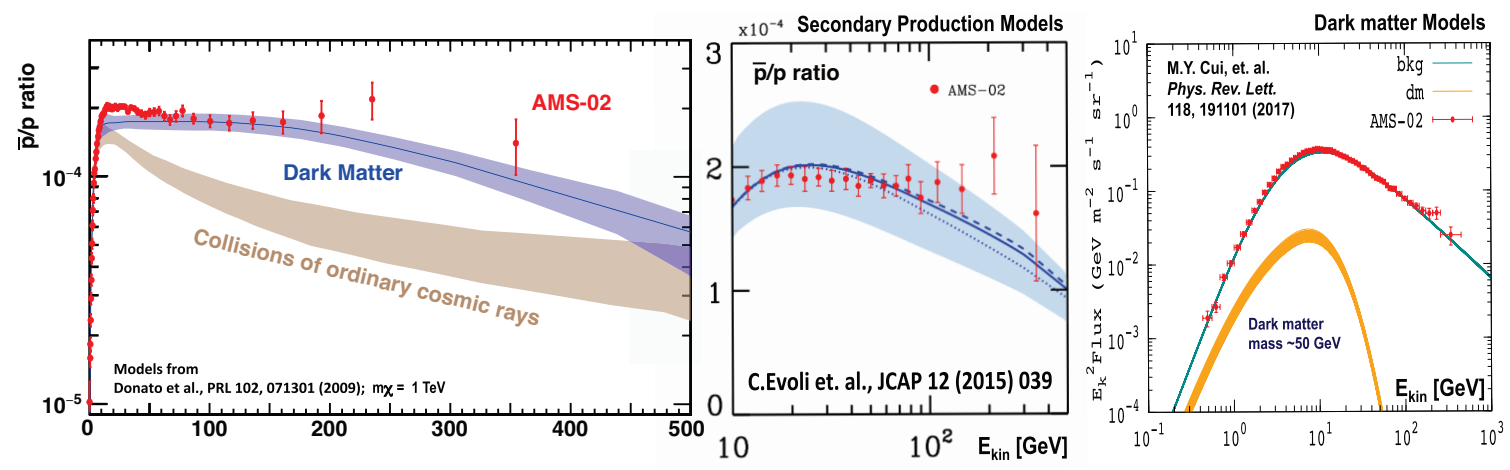

Figure 9: (left plot) The AMS results on the ratio $\bar{p} / p$ in comparison with the model predictions prior to AMS that expected decreasing ratio secondary antiprotons with rigidity. The AMS measurement extends the energy range to $450 \mathrm{GeV}$ and demonstrates that above $\sim 60 \mathrm{GeV}$ the $\bar{p} / \mathrm{p}$ remains almost flat. (middle and right plots) AMS data in comparison with the recent models of secondary antiproton production (middle plot) and Dark Matter antiproton production (right plot).

of the antiproton signal and the background in the $\left(\beta_{\mathrm{RICH}}-\Lambda_{\mathrm{TRD}}\right)$ plane for the absolute rigidity range $5.4-6.5 \mathrm{GV}$.

Overall, in the absolute rigidity range $1-450 \mathrm{GV} 3.49 \times 10^{5}$ antiproton events are selected. It is important to note that in the high rigidity range above $100 \mathrm{GV}$ AMS has 2200 events $[25,26]$. This can be compared with 3 events detected before AMS [27, 28]. The measured ratio $\bar{p} / p$ is presented in Fig. 9 together with model predictions. Above $\sim 60 \mathrm{GeV}$ the ratio is found to be independent on rigidity $[25,26]$. This observation caused a major revision of modeling cosmic ray antiprotons produced in ordinary collisions of protons with interstellar media as seen from comparison of the corresponding curves in Fig. 9 on the left plot [29] (done in 2009) and the middle [30] and right [31] plots (done in 2015 and 2017 for the middle and right plots, respectively). Still, even revised conservative models (with generously assigned theoretical uncertainties [30]) predict $30-40 \%$ drop of the ratio from the maximum, not supported by the AMS data, whereas optimistic models (with tight uncertainties for secondary antiproton production) see quite a bit of discrepancies with experimental data, ascribing these discrepancies to the Dark Matter contribution [31].

It is interesting to compare behavior of the spectra of all elementary particles among themselves. Traditionally, electrons and protons are assumed to be primary cosmic rays, i.e. particles produced directly at sources of cosmic rays like exploding supernovae. On the contrary, positrons and antiprotons are assumed to be secondary cosmic rays, i.e. coming from the interaction of primary cosmic rays with the interstellar media. In addition, electrons and positrons have much smaller mass than protons and antiprotons so they lose much more energy in the galactic magnetic fields. This is illustrated in Fig. 10, where the behavior of the electron spectrum is compared with that of the proton spectrum. As expected, in the rigidity region free of solar modulation effects, above $20 \mathrm{GeV}$, the proton spectrum is much harder that the electron spectrum (see Fig. 10a). However, further studies by AMS bring a lot of surprises. As seen in Fig. 10b, the behavior of antiprotons (assumed to be secondaries) and protons (assumed to be primaries) at high rigidities are very similar, while the behavior of electrons (i.e. primaries) and positrons (secondaries) are very different as illustrated by the AMS data in Fig. 10c. Most surprising is that above $60 \mathrm{GeV}$, 

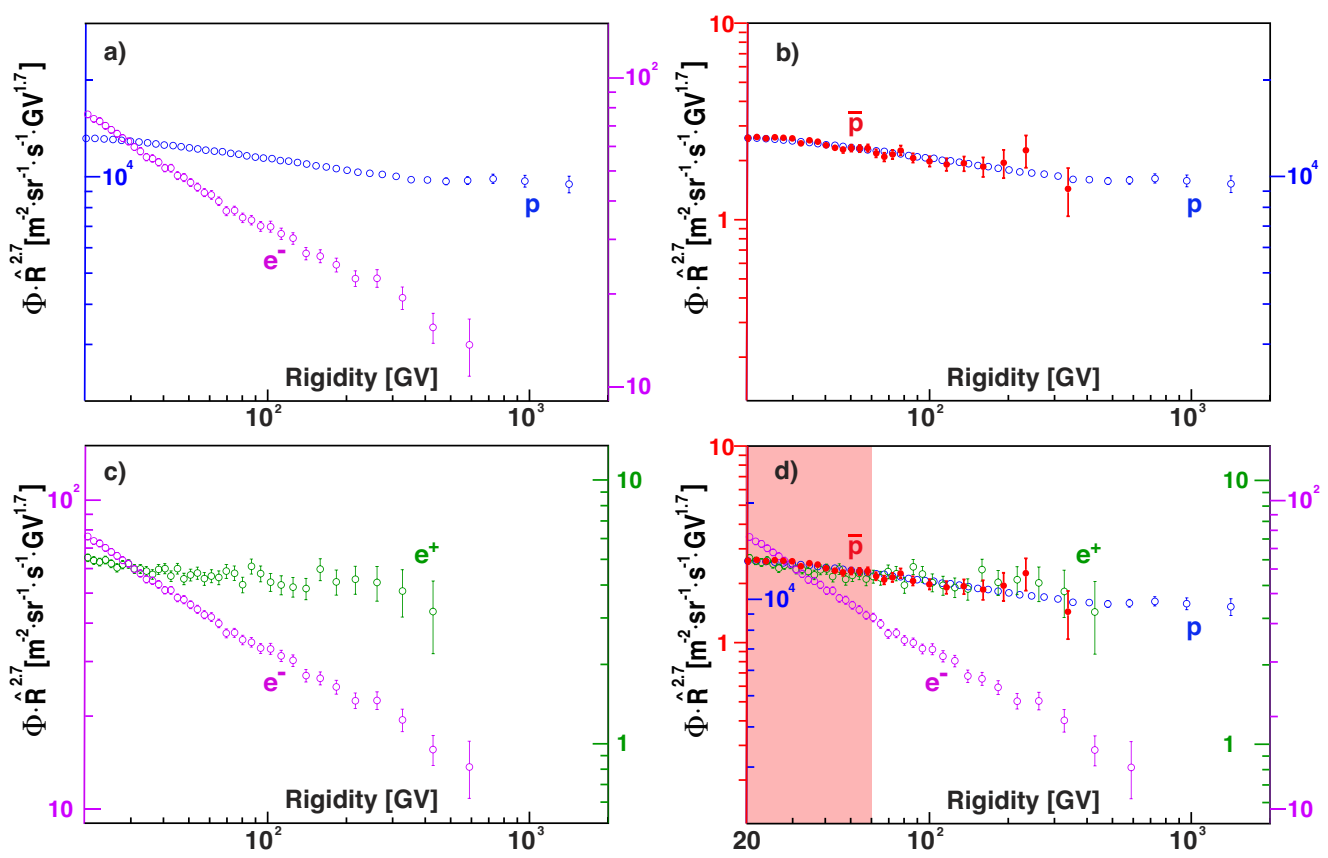

Figure 10: AMS measurements of the fluxes of elementary particles: (a) comparison of the functional behavior of the proton and electron fluxes; (b) comparison of the functional behavior of the proton and antiproton fluxes; (c) comparison of the functional behavior of the positron and electron fluxes; (d) the positron, antiproton and proton fluxes show the very same functional behavior in the absolute rigidity range 60-500 GV, whereas electrons exhibit distinctly different dependence.

positrons, protons and antiprotons display identical an energy dependence whereas electrons exhibit a totally different energy dependence as shown in Fig. 10d. The physics explanation for this AMS observation is yet to be found.

\section{Properties of fluxes of primary and secondary nuclei}

It is believed that protons, helium, carbon and oxygen are produced directly from primary sources in supernova remnants whereas lithium, beryllium and boron are produced from the collision of primary cosmic rays with the interstellar medium. Primary cosmic rays carry information about their original spectra and propagation, and secondary cosmic rays carry information about the propagation of primary and secondary cosmic rays and the interstellar medium.

Helium is the second most abundant cosmic ray. It is believed to be produced in astrophysical sources similar to those producing cosmic ray protons, and therefore its spectrum can be described by the very same power law function (see for instance the current PDG Chapter "29. COSMIC RAYS"). Similar to the measurement of protons, the first indication of more complex dependence came from the combined analysis of the AMS-01 and CREAM data [20, 21]. However, the detailed understanding of this behavior required the accuracy of the AMS detector [23, 32]. Fig. 11 (left plot) shows the AMS results on the helium flux as a function of rigidity together with the earlier results of PAMELA. Similarly to the proton spectrum, the helium spectrum measured by AMS 

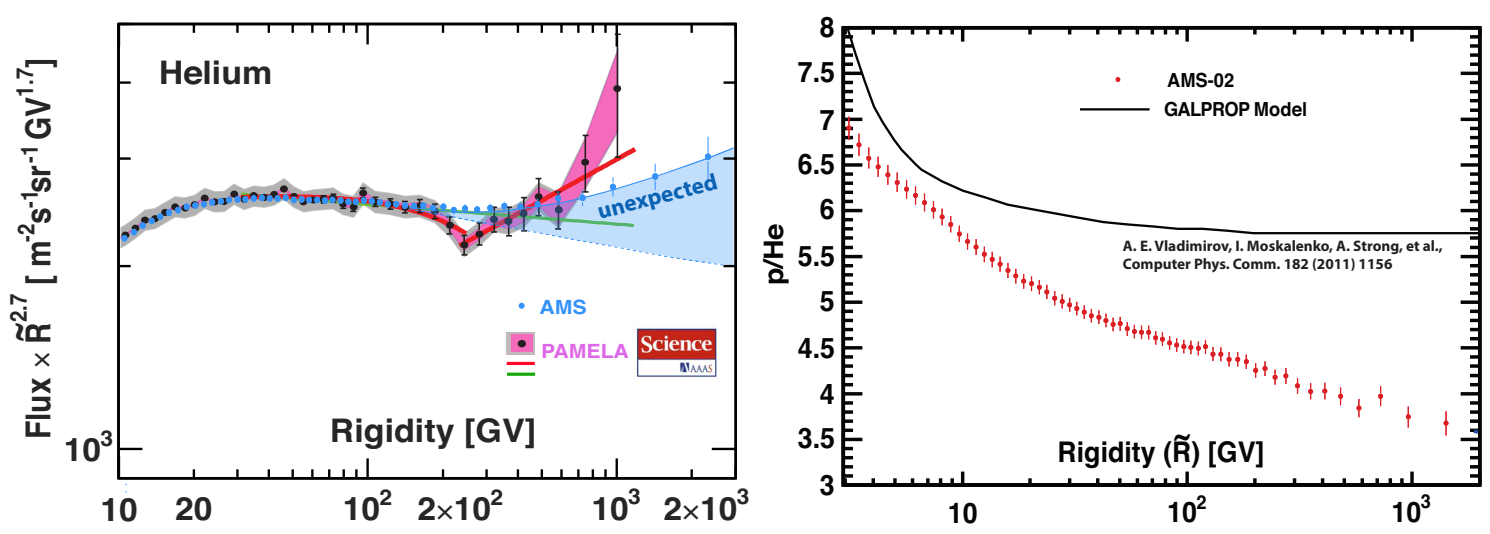

Figure 11: (left plot) The AMS helium flux (blue circles) hardens progressively from $100 \mathrm{GV}$ over the range of few hundred GV as indicated by the blue shaded area on the plot. Abrupt spectral hardening at 230-240 GV reported by the PAMELA experiment (also shown on the plot [24]) is not supported by the precision AMS data. (right plot) The proton-to-helium flux ratio showing a steep decease at high rigidities in contrast to flat theoretical predictions.

hardens progressively above $100 \mathrm{GV}$ over the range of few hundred $\mathrm{GV}$, and not very abruptly at 230-240 GV as show PAMELA results [24].

It is important to note that the ratio of proton-to-Helium fluxes is not constant at high rigidities, as traditionally assumed, but decreasing with rigidity as illustrated in Fig. 11 (right plot). With this observation, it was a big surprise to see that the ratios of other primary cosmic ray nuclei such as carbon and oxygen to helium are constant at high rigidities, as illustrated in Fig. 12 for the $\mathrm{C} / \mathrm{He}$ and $\mathrm{O} / \mathrm{C}$ ratios $[23,33]$.

In 2010 studying behavior of high energy primary cosmic nuclei (a combination of $\mathrm{C}, \mathrm{O}, \mathrm{Ne}$, $\mathrm{Mg}, \mathrm{Si}, \mathrm{Fe}$ ) CREAM noted that "A broken power law gives a better fit to our data." [21] (see also Fig. 13 (left plot)). The individual nuclei spectra measured by CREAM exhibit less conclusive behavior as illustrated in Fig. 13 (right plot) for the oxygen spectrum. AMS performes unique studies in this rigidity range to understand the dynamics of progressive hardening of the individual primary and secondary nuclei spectra. For instance, comparison of fluxes of carbon, nitrogen and
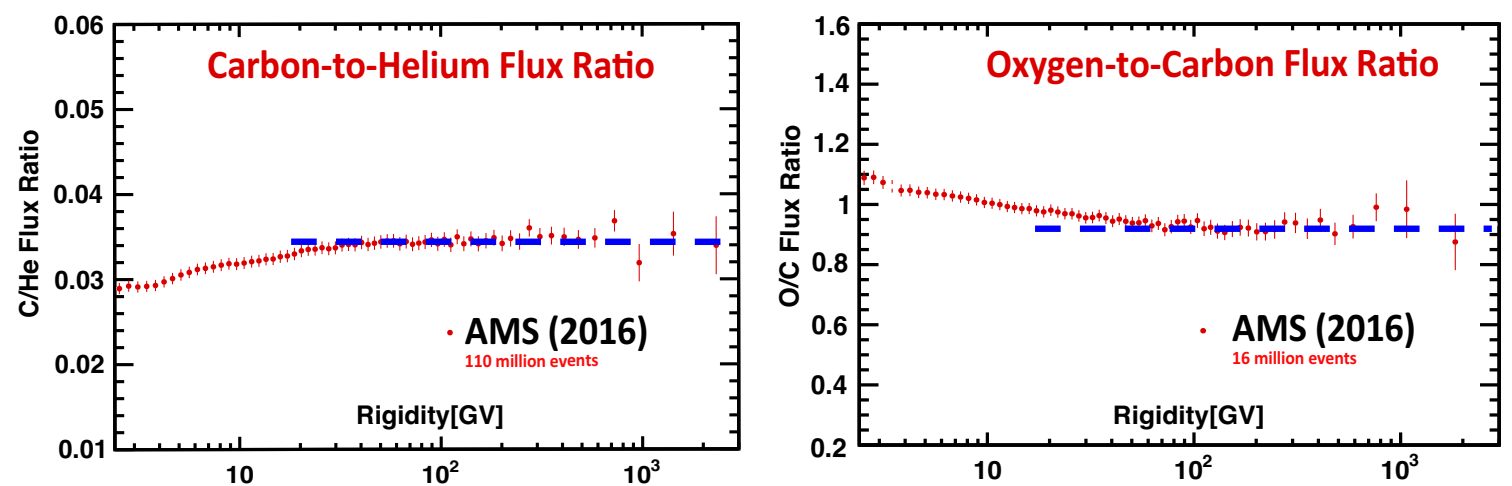

Figure 12: The carbon-to-helium (left) and oxygen-to-carbon (right) flux ratios. 

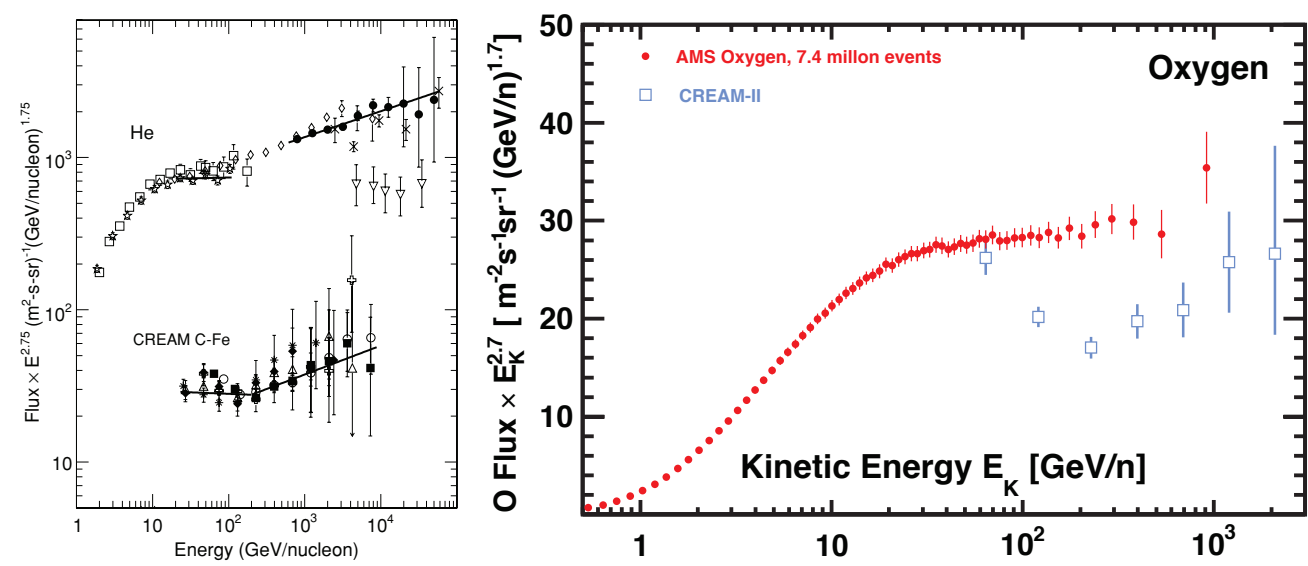

Figure 13: (left plot, taken from [21]) A fit of "broken power law" to combined CREAM data on C, O, Ne, $\mathrm{Mg}, \mathrm{Fe}$ nuclei. (right plot) Comparison of the AMS oxygen spectrum with the CREAM measurements [21] shows that conclusions on the dynamics of the oxygen spectrum hardening from these two measurements are different.

oxygen shows that above $60 \mathrm{GV}$ carbon and oxygen spectra show identical behavior to the spectrum of helium [33], whereas nitrogen flux is somewhat softer (see Fig. 14), indicating that nitrogen flux has both primary and secondary components [23].

Although lithium is assumed to be a secondary cosmic ray, surprisingly, its spectrum behaves similarly to protons and helium in that none of the three fluxes can be described by a single power law and they do change their behavior at the same rigidity as seen in Fig. 15.

Other secondary cosmic rays being measured by AMS include boron and beryllium. The unstable isotope of beryllium, ${ }^{10} \mathrm{Be}$, has a half-life of 1.5 million years and decays into $\mathrm{B}$. The $\mathrm{Be} / \mathrm{B}$ ratio therefore increases with energy due to time dilation when the $\mathrm{Be}$ approaches the speed of light. Hence, the ratio of beryllium to boron provides information on the age of the cosmic rays in the galaxy. As shown in Fig. 16, from this AMS has determined that the age of cosmic rays in the galaxy is 12 million years.

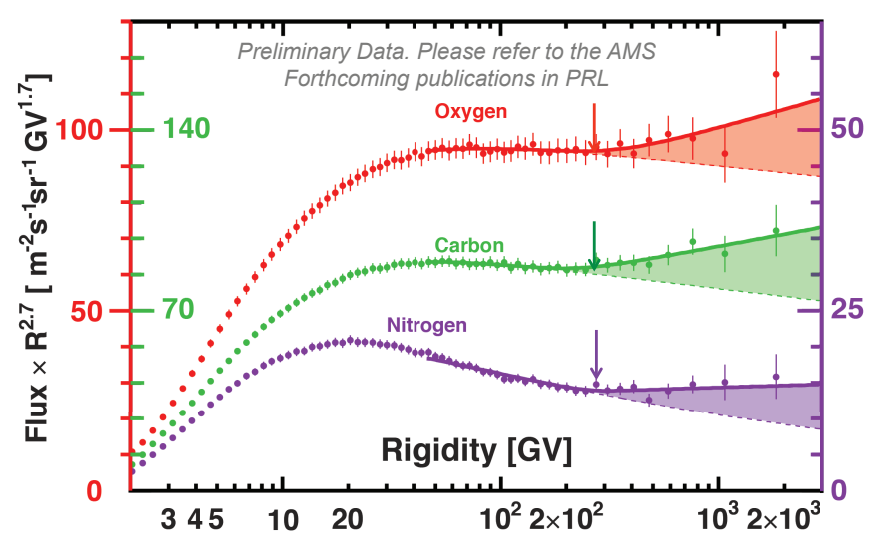

Figure 14: The spectra of carbon, nitrogen and oxygen all undergo a smooth transition to harder spectrum at around $300 \mathrm{GV}$. However nitrogen spectrum shows a different functional behavior. 


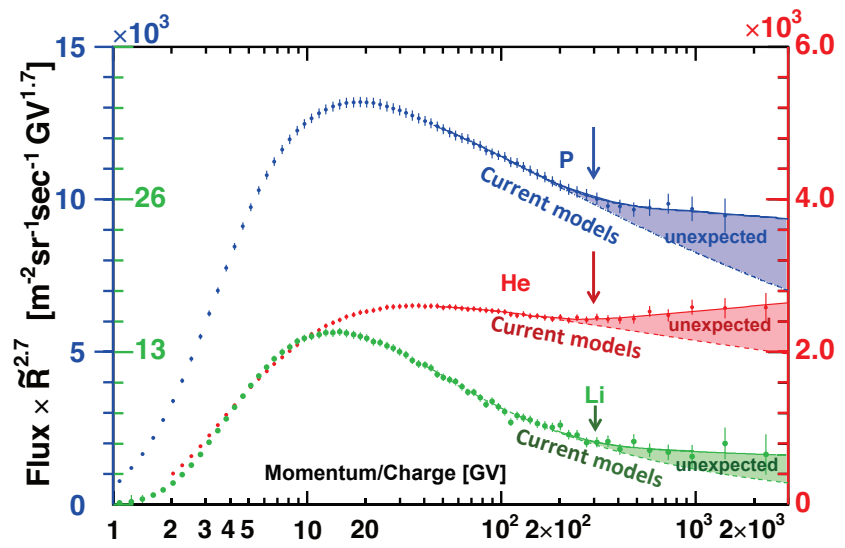

Figure 15: The spectra of protons, helium and lithium all undergo a smooth transition to harder spectrum at around $300 \mathrm{GV}$.

The flux ratio between secondaries (B) and primaries (C) provides information on propagation and the average amount of interstellar material (ISM) through which the cosmic rays travel in the galaxy. Cosmic ray propagation is commonly modeled as a fast moving gas diffusing through a magnetized plasma. Various models of the magnetized plasma predict different behavior. Remarkable, as shown in Fig. 17, above $65 \mathrm{GeV}$, the B/C ratio measured by AMS is well described by a single power law $\mathrm{B} / \mathrm{C}=R^{\delta}$ with $\delta=-0.333 \pm 0.015$ [34]. This is in agreement with the Kolmogorov turbulence model of magnetized plasma where $\delta=-1 / 3$ asymptotically. Of equal importance, the $\mathrm{B} / \mathrm{C}$ ratio does not show any significant structures in contrast to many cosmic ray models.
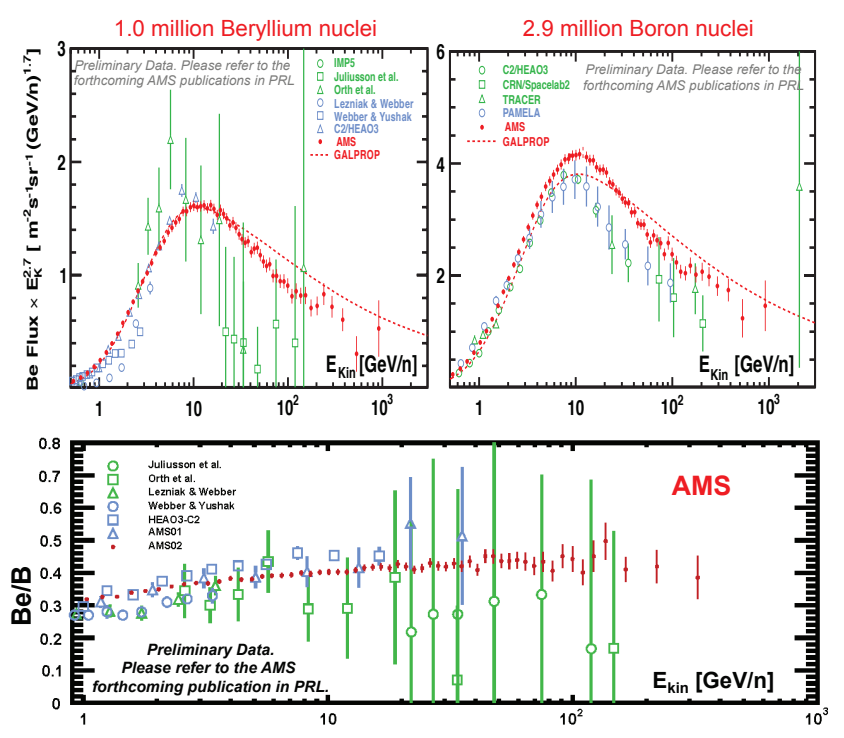

Figure 16: Beryllium (top left plot) and boron (top right plot) fluxes show significant improvements in the accuracy and energy reach in comparison to the previous measurements. (bottom plot) The beryllium-toboron $(\mathrm{Be} / \mathrm{B})$ flux ratio increases with energy due to time dilation of the decaying Be. The measurement yields the age of cosmic rays in the galaxy of $\sim 12$ million years. 


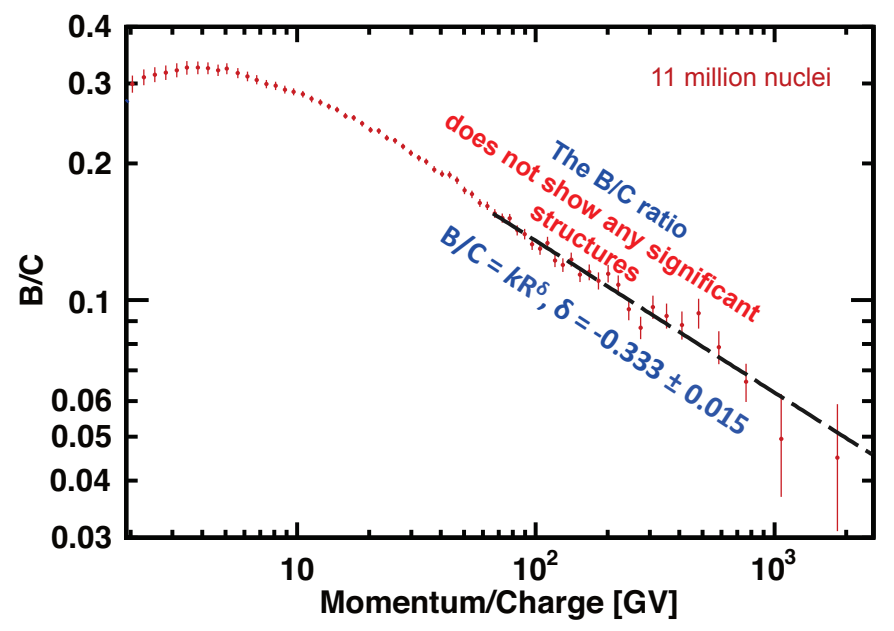

Figure 17: The B/C ratio does not show significant structures in contrast to many cosmic ray models requiring such structures at high rigidities. Above $65 \mathrm{GV}$, the $\mathrm{B} / \mathrm{C}$ ratio is well described by a single power law.

\section{Complex Antimatter in Cosmic Rays}

The Big Bang origin of the Universe requires that matter and antimatter be equally abundant at the very hot beginning of the universe. The search for the explanation for the absence of antimatter in a complex form is known as Baryogenesis. Baryogenesis requires both a strong symmetry breaking and a finite proton lifetime. Despite the outstanding experimental efforts over the last half a century, no evidence of strong symmetry breaking nor of proton decay have been found. Therefore, the observation of a single anti-helium event in cosmic rays is of great importance.

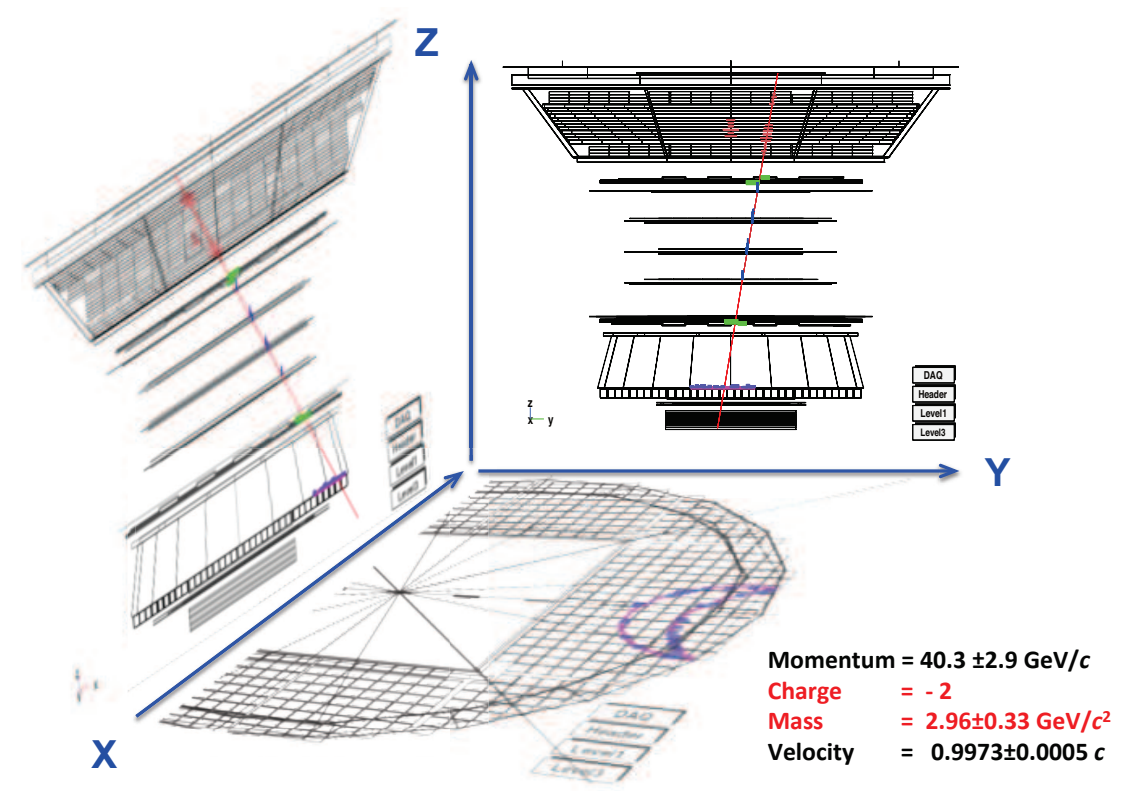

Figure 18: An antihelium candidate. 
In six years, AMS has collected 100 billion events. From this unparalleled sample of charged cosmic rays 700 million events with charge $|Z|=2$ are selected. All these events have been identified as Helium nuclei, except few that have negative rigidity or $Z=-2$. One of these anti-He candidates is presented in Fig. 18. All of the $\mathrm{Z}=-2$ candidates have one common and peculiar feature - mass around ${ }^{3} \mathrm{He}$.

At a rate of approximately one antihelium candidate per year and a required signal to background rejection of one in a billion, a detailed understanding of the instrument is required but is exceedingly difficult and time consuming. In the coming years one of our main efforts is to perform stringent detector verification and to collect more data in order to ensure that these $Z=-2$ events are indeed anti-helium.

\section{Conclusions}

In six years on the ISS, AMS has recorded more than 100 billion cosmic ray events. The latest AMS measurements of the positron spectrum and positron fraction, the antiproton/proton ratio, the behavior of the fluxes of electrons, positrons, protons, helium and other nuclei provide precise and unexpected information on the production, acceleration and propagation of cosmic rays. The accuracy and characteristics of the data, simultaneously from many different types of cosmic rays require the development of a comprehensive model of cosmic rays.

Of particular significance is our study of complex antimatter in the cosmos. Through stringent detector verification, collecting additional data and anti-deuteron analysis we will ensure that the observed $\mathrm{Z}=-2$ events are indeed anti-helium .

As a magnetic spectrometer studying cosmic rays, AMS is unique in its precision and energy reach. For the foreseeable future this is the only magnetic spectrometer in space to perform precision measurements and to explore the unknown with high expectations for exciting discoveries.

\section{References}

[1] A. Kounine, Int. J. Mod. Phys. E 21 (2012) 123005; S.C.C. Ting, Nucl. Phys. B, Proc. Suppl. 243-244 (2013) 12.

[2] B. Alpat et al., Nucl. Instrum. Meth. A 613 (2010) 207; G. Ambrosi et al., Nucl. Instrum. Meth. A 869 (2017) 29.

[3] V. Bindi et al., Nucl. Instrum. Meth. A 743 (2014) 22.

[4] B. Roe et al., Nucl. Instrum. Meth. A 543 (2005) 577.

[5] F. Hauler et al., IEEE Trans. Nucl. Sci. 51 (2004) 1365; P. Doetinchem et al., Nucl. Instrum. Meth. A 558 (2006) 526; Th. Kirn, Nucl. Instrum. Meth. A 706 (2013) 43.

[6] M. Aguilar et al., Phys. Rev. Lett. 110 (2013) 141102; L. Accardo et al., Phys. Rev. Lett. 113 (2014) 121101.

[7] M. Aguilar-Benitez et al., Nucl. Instrum. Meth. A 614 (2010) 237; F. Giovacchini, Nucl. Instrum. Meth. A 766 (2014) 57.

[8] C. Adloff et al., Nucl. Instrum. Meth. A 714 (2013) 147; A. Kounine, Z. Weng, W. Xu and C. Zhang, Nucl. Instrum. Meth. A 869 (2017) 110. 
[9] S. Agostinelli et al., Nucl. Instrum. Meth. A 506 (2003) 250; J. Allison et al., IEEE Trans. Nucl. Sci. 53 (2006) 270.

[10] M. Turner and F. Wilczek, Phys. Rev. D42 (1990) 1001; J. Ellis, $26^{\text {th }}$ ICRC Salt Lake City (1999) astro-ph/9911440; H. Cheng, J. Feng and K. Matchev, Phys. Rev. Lett. 89 (2002) 211301; S. Profumo and P. Ullio, J. Cosmology Astroparticle Phys. JCAP07 (2004) 006; D. Hooper and J. Silk, Phys. Rev. D 71 (2005) 083503; E. Ponton and L. Randall, JHEP 0904 (2009) 080; D. Hooper, P. Blasi and P. D. Serpico, JCAP 0901025 (2009) 0810.1527; Y-Z. Fan et al., Int. J. Mod. Phys. D19 (2010) 2011; M. Pato, M. Lattanzi and G. Bertone, JCAP 1012 (2010) 020.

[11] J. J. Beatty et al., Phys. Rev. Lett. 93 (2004) 241102.

[12] M. Aguilar et al., Phys. Lett. B 646 (2007) 145.

[13] O. Adriani et al., Nature 458 (2009) 607; O. Adriani et al., Astropart. Phys. 34 (2010) 1; O. Adriani et al., Phys. Rev. Lett. 111 (2013) 081102.

[14] M. Ackermann et al., Phys. Rev. Lett. 108 (2012) 011103.

[15] Z. Li on behalf of AMS, Presented at this conference, CRD079.

[16] M. Aguilar et al., Phys. Rev. Lett. 113 (2014) 121102; M. Aguilar et al., Phys. Rev. Lett. 113 (2014) 221102.

[17] Z. Weng on behalf of AMS, Presented at this conference, CRD065.

[18] There are many recent excellent theoretical articles on the positron fraction. A few examples are: K. Blum et al., Phys. Rev. Lett. 111 (2013) 211101; L. Bergström et al., Phys. Rev. Lett. 111 (2013) 171101; J. Kopp, Phys. Rev. D 88 (2013) 076013; I. Cholis and D. Hooper, Phys. Rev. D 88 (2013) 023013; T. Linden and S. Profumo, Astrophys. J. 772 (2013) 18; L. Feng et al., Phys. Lett. B 728 (2014) 250 .

[19] I. Gebauer on behalf of AMS, Presented at this conference, CRD071.

[20] J. Alcaraz et al., Phys. Lett. B 472 (2000) 215; J. Alcaraz et al., Phys. Lett. B 494 (2000) 193.

[21] H.S. Ahn et al., Astro. J. Lett. 714 (2010) L89.

[22] M. Aguilar et al., Phys. Rev. Lett. 113 (2014) 221102.

[23] V. Choutko on behalf of AMS, Presented at this conference, CRD058.

[24] O. Adriani et al., Science 332 (2011) 69.

[25] M. Aguilar et al., Phys. Rev. Lett. 117 (2016) 091103.

[26] W. Xu on behalf of AMS, Presented at this conference, CRD059.

[27] O. Adriani et al., Phys. Rev. Lett. 102 (2009) 051101.

[28] K. Abe et al., Phys. Rev. B 670 (2008) 103.

[29] F. Donato et al., Phys. Rev. Lett. 102 (2009) 071301.

[30] G. Giesen et al., JCAP 09 (2015) 023; R. Kappl et al., JCAP 10 (2015) 034; C. Evoli et al., JCAP 12 (2015) 039.

[31] M.Y. Cui et al., Phys. Rev. Lett. 118 (2017) 191101; A. Cuoco et al., Phys. Rev. Lett. 118 (2017) 191102.

[32] M. Aguilar et al., Phys. Rev. Lett. 115 (2015) 211101.

[33] Q. Yan on behalf of AMS, Presented at this conference, CRD061.

[34] A. Oliva on behalf of AMS, Presented at this conference, CRD062. 